\title{
SUPERNOVA MECHANISMS: BEFORE AND AFTER SN1987a
}

\author{
Sidney H. Kahana \\ Physics Department \\ Brookhaven National Laboratory \\ Upton NY 11973 \\ U S A
}

\begin{abstract}
The impact of SN1987a on theoretical studies of the specific mechanism generating Type II supernovae is examined. The explosion energy extracted from analysis of the light curve for SN1987a is on the edge of distinguishing between a prompt explosion from a hydrodynamic shock and a delayed, neutrino-induced, explosion. The detection of neutrinos from 1987a is also reanalysed.
\end{abstract}

\section{Introduction}

The observation of Supernova (Shelton) 1987a $[1,2]$ on February 23 of this year in the Large Magellanic Cloud, has provided astrophysicists and neutrino-physicists with a magnificent opportunity to test out their equipment and theories. Some surprise was clearly created, especially for the presupernova evolvers who had not seriously expected blue giants to explode; on the other hand, the neutrino observations might be said to be just what one might expect for a gravitational collapse, Type II supernova (SNII). It is the collapse and explosion phase for such a supernova that concerns me here. I wish to report on new calculations [3] by my colleagues and myself, and to remind you of our earlier description [4] of the prompt mechanism for Type IIs. I will also present results [5] from the analysis of the neutrinos seen by Kamiokande (KII) [6] and by IMB [7].

There is a long history [8-11] of the difficulties inherent in producing a prompt explosion in an SNII progenitor, first thought to be red supergiants and now clearly also blue giants. It appeared early on that the analysis of the SN1987a light curve would help to distinguish between possible mechanisms. This may still prove to be correct. For the moment, however, both Nomoto and coworkers [12], as well as Woosley and coworkers [13], and others [14], have analysed the light curve and have arrived at explosion energies which may conceivably be generated by either a prompt or a delayed shock mechanism. The energies suggested, at this Symposium, from total luminosities, are 1.0 to $1.5 \times 10^{51} \mathrm{ergs}[12]$ and 0.5 to $1.5 \times 10^{51} \mathrm{ergs}$ [13], whose lower limits are perhaps too low to rule out delayed explosions. There remain problems, however. Nomoto and co-workers [12] finds an energy of as much as $2.5 \times 10^{51}$ ergs may be demanded by consistency with the colour temperature, while their description of the later time light curve analysis (from

This work has been supported by the U. S. Department of Energy under contract no. DEAC02-76CH00016. 
75 days to 125 days) is probably improved by increasing the ejected mass $\left(M_{E}\right)$ and thus also the explosion energy $\left(E_{\text {expl }}\right)$. There is always a degree of ambiguity associated with $M_{E}$ and $E_{\text {expl }}$, for example the initial velocities and initial propagation time for the shock to reach the photosphere are given by [12]

$$
t_{\text {prop }} \propto R_{0}\left(\frac{M_{E}}{E_{\text {expl }}}\right)^{1 / 2} .
$$

Increasing the mass at the same time as mixing the radioactive sources throughout the star will both keep the peak in the light curve near the observed 90 day point after explosion, and broaden the curve. Both of the features would improve the fit in Ref. 12 to the observed light curve.

There are two presently extant mechanisms for producing explosions in the collapsing core of SNII progenitors. In a prompt explosion the hydrodynamic shock produced just after bounce is sufficiently energetic to expel the stellar mantle and envelope. In a so-called delayed explosion a stalled, accretion shock is resuscitated by neutrino emission from a hot interior. Wilson, Mayle and Bethe [15] have discussed the neutrino-induced mechanism and in general produce low explosion energies $\$ 1.0 \times 10^{21}$ ergs for progenitor main sequence masses initially below $25 \mathrm{M}_{\odot}$. Baron, Cooperstein and Kahana were the first to generate successful prompt explosions for Woosley, Weaver $[16,17]$ models and can obtain explosion energies up to 2.5 or $3.0 \times 10^{51}$ ergs. These energies include corrections from nuclear burning in the mantle and from the gravitational binding of the mantle and envelope.

Perhaps the most novel aspect of SN1987a is the detection [6,7] of neutrinos from the production and cooling of a compact remnant. One hopes this is only the beginning of a new field of astronomy. The analysis I present here [5], parallel to the analysis of many other authors [23-28], finds remnant binding energy $\sim 2.0 \pm 0.5 \times 10^{53}$ ergs and remnant mass 1.2 to $1.7 \mathrm{M}_{\odot}$ consistent with what one expects for neutron star generation. An upper limit of $10-15 \mathrm{eV}$ may also be inferred for the electron neutrino mass.

\section{Elements in the Success of a Prompt Explosion}

\section{A. Core characteristics}

Theoretical modelling of the brief period from fuel exhaustion to collapse, bounce and shock formation in SNIIs is one of the more difficult problems in astrophysics. Early hydrodynamic simulations [8] at the beginning of this decade found that neutrino and dissociation losses stalled the shock inside the "iron" core, and that eventually accretion of mantle material would drive the core towards a black hole. An important factor in this demise of the hydrodynamic shock was the excessive core mass, $M_{\text {core }} \sim 1.50 \mathrm{M}_{\odot}$ to $1.55 \mathrm{M}_{\odot}$, predicted in presupernova modelling of massive stars. The Chandrasahkar limit $M_{C h} \sim 5.7 Y_{e}^{2}$ pretty well determines the core mass at collapse, but details of the presupernova calculations have led to an evolution of $M_{\text {core }}$ from the early Woosley, Weaver, Zimmerman [16] value 1.51 $\mathrm{M}_{\odot}$ to later Woosley, Weaver [17] level of $M_{\text {core }} \sim 1.36 \mathrm{M}_{\odot}$ for a star with the main sequence masses $M_{m s} \sim 12,15 \mathrm{M}_{\odot}$, and more recently to $M_{\text {core }} \approx 1.18 \mathrm{M}_{\odot}$ for a Nomoto-Hashimoto [18] model with main sequence mass $13 \mathrm{M}_{\odot}$. Should initial core masses at collapse stay as low as those in these recent calculations, prompt explosions will be hard to avoid and may obtain for progenitors in mass up to perhaps $20 \mathrm{M}_{\odot}$, i.e., for helium cores in the neighborhood of $6 \mathrm{M}_{\odot}$.

The core characteristics do not change appreciably in these diverse models with central densities near $10^{10} \mathrm{~g} / \mathrm{cm}^{3}$, a central electron fraction $Y_{e}^{c} \approx 0.42$, and temperature $T_{c} \approx 0.5 \mathrm{MeV}$. The central entropy per baryon rises and falls with the core mass, with high values inimical to healthy shocks. 
B. Background to Calculations: Input Physics To understand the delicacy of the collapse simulation, one should recall the virial theorem for the non-relativistic core matter supported by a relativistic electron gas, for which

$$
E_{\text {gravitational }}+E_{\text {internal }} \approx 0 \text { for } P \sim \rho^{4 / 3} .
$$

The adiabatic collapse preserves this relation but drives the total gravitational energy to above $10^{53}$ ergs. Thus, the shock energy for a viable prompt explosion, $\sim 2 \times 10^{51} \mathrm{ergs}$, is small in comparison to the internal or gravitational energy. Simulation must be accurate both in computation and in the input physics.

A second important feature to keep in mind is the small radius of the homologously collapsing core at maximum density, $R_{\text {core }} \approx 15 \mathrm{fm}$, in comparison to the Schwarzschild radius $R_{\mathrm{s}} \approx 2 \mathrm{~km}$ for the 0.7 or so solar masses inside this core. The sensitivity in physical effects determining the final shock energy extends also to the treatment of gravitation. General relativity seems necessary, both helps and hurts the prompt mechanism, but in the end is crucial to producing explosions.

The elements of physics input that are marked out for careful treatment, then, include:

1. $\beta$-capture, neutrino transport: effect on $Y_{e}$.

2. Hadronic equation of state at high density, $\rho=\rho_{0}$ to $4 \rho_{0}, \rho_{0} \approx 2.4 \times 10^{24} \mathrm{~g} / \mathrm{cm}^{3}$ the saturation density for the asymmetric nuclear matter found in the collapsing core. A moderate softening of nuclear matter under these conditions allows the core to collapse further into the gravitational well and thus increases the shock energy at formation.

3. General relativity magnifies this effect, but at the same time reduces the mass of the homologous core at bounce and forces the shock to unfavourably traverse more stellar material on its way out of the core. The equation of state used in our astrophysical simulations has the simple form [19]

$$
P_{N}(\rho)=\frac{K_{0}[Z / A] \rho_{0}[Z / A]}{9 \Gamma}\left[\left(\frac{\rho}{\rho_{0}}\right)^{\Gamma}-1\right]
$$

for the cold, hadronic pressure. The incompressibility $K_{0}$ (and density $\rho_{0}$ ) are, of course, functions of the charge to mass ratio $Z / A$, the latter reduced by $\beta$-capture from 0.42 to nearer 0.32 in the collapse environment. A good measure of the softening so helpful to the prompt mechanism then follows from the behaviour of the incompressibility in asymmetric matter $(N=2 Z)[19,4]$

$$
K_{0}(Z / A)=K_{0}^{s y m m}\left[1-2\left(\frac{Z}{A}-\frac{1}{2}\right)^{2}\right] .
$$

The combination of a softer equation of state with relativistic gravitation is the key to the prompt mechanism.

4. Laboratory constraints on the hadronic equation of state do exist and must be respected.

a. The incompressibility at normal nuclear density $\rho_{0}$ is reasonably well extracted from the energy of the breathing mode in heavy nuclei to be [20]

$$
K_{0}^{\text {symm }}=210 \pm 30 \mathrm{MeV} \text {, at } Z / A=1 / 2 .
$$

b. In contrast, early analysis of relativistic heavy ion experiments at LBL [21] pointed to a considerably stiffer nuclear matter at high density. This is still an open question, but further investigations [22] suggest the neglect in the analysis of heavy ion collisions of the velocity (and 
hence density) dependence of nuclear forces as the culprit in the discrepancy between heavy ion collisions and breathing mode equations of state.

\section{Results of Prompt Mechanism Simulations}

The parameters of explosions resulting from our hydrodynamic simulations have been extensively reported. Confirmation of these results are seen in the recent work of Bruenn [23], some of which is as yet unpublished. A key component of any such calculations is the $\nu$-transport scheme which in our calculations has only recently been carried out in complete detail to the level found in Bruenn [23] or Wilson and Mayle [15].

It suffices to quote some results (Table I) for the $12,15 \mathrm{M}_{\odot}$ initial models of Woosley-Weaver and for the $13 \mathrm{M}_{\odot}$ model of Nomoto-Hashimoto. The much lower core masses found by the latter group for even $20 \mathrm{M}_{\odot}\left(\mathrm{M}_{\mathrm{He}} \approx 6.0 \mathrm{M}_{\odot}\right)$ progenitors presage viable explosions in these cases as well. A vital factor in our introduction of full transport is once again the strong role played by symmetry effects. A high symmetry energy suppresses free proton number (and hence $\beta$-capture) in the collapse environment and thus sustains a high $Y_{e}$ throughout the collapsing core. This in turn leads to shock formation at a favourably large radius.

From Table I one can conclude that within reasonable ranges for $K_{0}(Z / A)$ and the adiabatic index $\Gamma$, prompt explosion energies can describe SN1987a or perhaps even more energetic supernovae. A close examination of the work of Shigeyama, Nomoto and Hashimoto [12] leads me to suspect they may have underestimated the energy in 1987a.

\section{Neutrino Detection}

The 'sightings' of simultaneous, bunches of neutrinos in the KII [6] and IMB [7] detectors some three or four hours before optical observations of SN1987a is surely as good a demonstration of the existence of gravitational collapse supernovae as we can desire. The very short time between neutrinos and optical visibility is a surprise, speaking to the small size and unusual nature of the progenitor. We have performed [5] one of the many parallel analyses of these neutrinos [23-28].

At the heart of our analysis are the equations

$$
\begin{aligned}
\frac{d E}{d t} & =L=(0.011)\left(\frac{T}{M e V}\right)^{4}\left(\frac{R}{10 \mathrm{~km}}\right)^{2} \times 10^{51} \mathrm{ergs} / \mathrm{sec} . \\
T & =T_{0} e^{-t / \tau}
\end{aligned}
$$

describing the compact remnant emission as black body from a sharp neutrinosphere and with Newtonian cooling of the remnant. The analysis must take account of the neutrino cross sections and detector efficiencies [5].

The observed mean energy more or less determines the initial cooling temperature $T_{0}$; the total number of neutrinos in the detectors then determine the binding energy $B$ and compact remnant mass $M_{n s}$. The chronology of detected events, divorced from their energy distribution, yields a cooling time $\tau$. We find an acceptable range for $T_{0}$ between 4.5 and $5.5 \mathrm{MeV}$ and then extract $B=2.0 \pm 0.5 \mathrm{MeV}$ and $M_{n s}=1.2$ to $1.6 \mathrm{M}_{\odot}$. Further, there is a complete consistency in the numbers extracted separately from $\mathrm{KII}$ and $\mathrm{IMB}$ data within the stated range of initial temperature.

Finally, I turn to the question of limits on neutrino masses. One can obtain a good limit on $m_{D_{e}}$ by examination of Table II, constructed by mapping the Kamiokande data back to its source at the supernova, after imposition of a finite mass. The KII data itself corresponds to $m_{\nu_{e}}=0$; I 
Table I Explosion Energies from the Prompt Mechanism, for Various Collapse Models taken from Refs. 3 and 4. Equation of state parameters $K_{0}^{\text {symm }}, \Gamma$ are the equivalent incompressibility at saturation for symmetric matter $Z / A=1 / 2$, and the adiabatic index, as given in equations (3) and (4). All calculations take full account of general relativity except for model \#38*, which is Newtonian. The maximum central density reached in the calculation $\rho_{\max }^{c}$ is in units of the saturation density appropriate to the asymmetric matter $Z=1 / 3$ relevant to bounce, which is $\rho_{0}(1 / 3)=2.4 \times 10^{14} \mathrm{~g} / \mathrm{cm}^{3}$. The precollapse models for \#48-45 are the main sequence $M=$ $12,15 \mathrm{M}_{\odot}$ models of Ref. 17, while \#61-63 are from the $13 \mathrm{M}_{\odot}$ model of Ref. 18. The explosion energy $E_{\text {expl }}$ was obtained from the estimated shock energy by correcting for oxygen burning in the mantle and gravitational binding of mantle and envelope. Models are further distinguishable by the symmetry energy $W_{s}$ which we believe is experimentally closer to the higher values in the table and by a trapping density which is set at $0.4 \times 10^{12} \mathrm{~g} / \mathrm{cm}^{3}$ in the first six models in the table, and at the more realistic $1 \times 10^{12} \mathrm{~g} / \mathrm{cm}^{3}$ for \#62, 63 .

\begin{tabular}{|c|c|c|l|l|r|c|}
\hline Model number & Mass & $K_{0}^{\text {symm }}$ & $\Gamma$ & $W_{\diamond}$ & $\frac{\rho_{\text {max }}^{c}}{\rho_{0}(0.33)}$ & $E_{\text {expl }}$ \\
\hline & $\mathrm{M}_{\odot}$ & $\mathrm{MeV}$ & & $\mathrm{meV}$ & & $10^{51}$ ergs \\
\hline $38^{*}$ & 12 & 180 & 2 & 29.3 & 2.3 & 0.1 \\
40 & 12 & 180 & 2 & 29.3 & 12.0 & 3.2 \\
41 & 12 & 180 & 3 & 29.3 & 3.1 & 0.8 \\
43 & 15 & 180 & 2.5 & 29.3 & 4.1 & 1.7 \\
45 & 15 & 90 & 3 & 29.3 & 4.0 & 0.8 \\
\hline 61 & 13 & 180 & 2.5 & 29.3 & 4.1 & 2.4 \\
62 & 13 & 180 & 2.5 & 36.0 & 4.1 & 2.6 \\
63 & 13 & 180 & 2.5 & 34.0 & 4.1 & 1.9 \\
\hline \hline
\end{tabular}

have also included the mapping for $m_{D_{e}}=10$ and $m_{D_{e}}=15 \mathrm{eV}$. It is my opinion that the first eight KII events in $1.92 \mathrm{sec}$. constitute the 'real' cooling pulse. The width in time of this pulse starts at $1.92 \mathrm{sec}$. and is stretched to $5.4 \mathrm{sec}$. for a $10 \mathrm{eV}$ mass, to $10.8 \mathrm{sec}$. for a $15 \mathrm{eV}$ mass. Moreover, the spectrum itself becomes very hard for either of these masses, with neutrinos of low energies being emitted well before those of high energies as the neutron star cools. To select a mass of $30 \mathrm{eV}$ and claim this is a more conservative [28] limit for the neutrino mass seems unreasonable; one is then dealing with almost unconnected events at the supernova. The energy versus time sequencing and pulse width become unacceptable near $m_{D_{e}}=15 \mathrm{eV}$, especially since the source pulse width is then $\sim 10$ sec. somewhat larger than the cooling time $\tau / 4 \leqslant 4$ sec. we find from the data itself.

The above limit is comparable to or slightly better than that obtainable from the presently best laboratory experiments [29]. How does one evaluate this estimate statistically? The weakest link in the argument is I think the dependence of the mapped pulse width on the time of arrival of the lowest energy event \#3. The probability that both \#3 and \#6 (rejected as background) are background events determines the level of confidence in our conclusions. This probability is roughly $5 \%$. Otherwise, one would rely on event \#4 (9.5 MeV electron energy) and extract a limit closer to $20 \mathrm{eV}$ for the mass upper bound.

The late time $\mathrm{KII}$ events, after 10 seconds, are potentially quite interesting. It would seem the probability of all three of these events \#10,11, 12 being background is slight [6]. They could represent some reheating of the compact remnant due to, say, material falling back into the core or, more speculatively, a phase change in the core. However, the falling back of matter not ejected in the explosion should not take as long as 10 seconds. 
Table II Limit on the neutrino mass. The KII event sequence is mapped back in time to the source as a function of neutrino mass. The pulse width for the first eight events becomes increasingly long as the neutrino mass $m_{D_{e}}$ increases, and the spectrum becomes increasingly hardened. Knowledge of the supernova and neutron star cooling strongly suggest a mass of $15 \mathrm{eV}$ as an upper limit.

\begin{tabular}{|c|c|c|c|c|}
\hline Event Number & $t_{\text {detection }}$ (secs) & Energy $(\mathrm{MeV})$ & $\left(t-t_{1}\right)$ source & $\left(t-t_{1}\right)$ source (secs) \\
\hline 1 & & & $\frac{m_{\nu_{e}}=10 \mathrm{eV}}{0}$ & $\frac{m_{\nu_{e}}=15 \mathrm{eV}}{0}$ \\
2 & 0.0 & 20 & -0.63 & -1.55 \\
3 & 0.107 & 13.5 & -3.50 & -8.27 \\
4 & 0.303 & 7.5 & -2.00 & -4.90 \\
5 & 0.324 & 9.2 & -0.38 & -1.49 \\
\hline 6 & 0.507 & 12.8 & & \\
(rejected) & 0.686 & 6.5 & & 1.97 \\
\hline 7 & 1.541 & 35.4 & 1.80 & 1.89 \\
8 & 1.728 & 21.0 & 1.92 & 1.89 \\
9 & 1.915 & 19.8 & 6.45 & 7.39 \\
10 & 9.2 & 8.6 & 9.55 & 8.49 \\
11 & 10.4 & 13.0 & 9.87 & 6.71 \\
12 & 12.4 & 8.9 & & 10.8 \\
\hline Pulse width & & & & \\
at source & 1.92 & & & \\
(first 8 events) & & & & \\
\hline \hline
\end{tabular}

There are a number of other interesting limits to be drawn on neutrino properties by somewhat more sophisticated use of the supernova dynamics. Putting another neutrino-antineutrino pair [30], i.e., another two species, into any calculation of the neutron star cooling would probably accelerate this process unacceptably. Further, one can place an upper limit [5] of $45 \mathrm{eV}$ on the mass of any species mixing with the electron neutrino, else no supernova mechanism would succeed, delayed or prompt.

\section{References}

1. I. Shelton (Las Campanas Observatory, Chile): International Astronomical Circular No. 4316, 24 February 1987, communicated by W. Kunkel and B. Madore.

2. R. H. McNaught: IAU Circular No. 4389 (1987);

R. Catchpole, et al: M.N.R.A.S. (submitted 1987);

R. Gilmossi, et al: Nature 328, 318 (1987);

R. Kirshner, G. Sonneborn, D. M. Crenshaw, and G. E. Nassiopoulos: Astrophys. J. Lett. (in press).

3. E. Baron, H. A. Bethe, G. E. Brown, J. Cooperstein, and S. Kahana: Phys. Rev. Lett. 59, 736 (1987).

4. E. Baron, J. Cooperstein, and S. Kahana, Phys. Rev. Lett. $\underline{55}$, 126 (1985).

5. S. Kahana, in Proceedings of "La Structure Elementaire de la Matiere," (Les Houches, France, March 1987);

S. Kahana, J. Cooperstein, and E. Baron: Phys. Lett. (in press).

6. R. Hirata et al: Phys. Rev. Lett. $\underline{58}, 1490$ (1987).

7. R. M. Bionta et al: Phys. Rev. Lett. $\underline{58}, 1494$ (1987).

8. T. Masurek, J. Cooperstein, and S. Kahana: in "Dumand ' $80^{\circ}$, edited by V. J. Stenger (Hawaii Dumand Center, Honolulu, 1981) and in "Supernovae: A Summary of Current Research,"edited by M. Rees and R. J. Stoneham (Reidel, Dordrecht, 1982). 
9. J. R. Wilson: Ann. N. Y. Acad. Sci. $\underline{336}, 358$ (1980).

10. W. D. Arnett: in "Supernovae: A Summary of Current Research," edited by M. Rees and R. J. Stoneham (Reidel, Dordrecht, 1982).

11. W. Hillebrandt: in "Supernovae: A Summary of Current Research." edited by M. Rees and R. J. Stoneham (Reidel, Dordrecht, 1982).

12. T. Shigeyama, K. Nomoto, M. Hashimoto, and D. Sugimoto: Nature (submitted April 1987); and in Proceedings of ESO Workshop on SN1987a, (Garching, July 1987); and proceedings of this Symposium.

13. S. E. Woosley, P. A. Pinto, and L. Ensman: Astrophys. J. (submitted April 1987); and proceedings of this Symposium.

14. W. Hillebrandt, P. Hötlich, J. W. Truran, and A. Weiss: Nature (submitted April 1987).

15. J. R. Wilson, in Numerical Astrophysics, eds. J. Centrella, J. Leblanc and R. Bowers (Jones and Bartlett: Boston 1985);

H. A. Bethe and J. R. Wilson: Astrophys. J. 295, 14 (1985);

R. Mayle: University of California at Berkeley, $\mathrm{PhD}$ thesis (1984)unpublished.

16. T. A. Weaver, B. Zimmerman, and S. E. Woosley: Astrophys. J. 225, 1021 (1978).

17. S. E. Woosley and T. A. Weaver: Bull. Am. Astr. Soc. 16, 971 (1984); and private communication.

18. K. Nomoto and M. Hashimoto: Prog. in Part. \& Nucl. Phys. 17, 2670 (1986); and private communication.

19. S. H. Kahana: Prog. in Part. \& Nucl. Phys. 17, 231 (1986);

S. H. Kahana: in Conf. Proc. Weak and Electromagnetic Interactions in Nuclei (Heidelberg, FRG, July 1986);

E. Baron, J. Cooperstein, and S. H. Kahana: Nucl. Phys. A440, 744 (1985).

20. J. P. Blaizot: Physics Reports 64, 171 (1980).

21. H. A. Gustafsson et al: Phys. Rev. Lett. $\underline{52}, 1590$ (1984);

J. W. Harris et al: Phys. Lett. 153,377 (1982).

22. T. Ainsworth, E. Baron, G.E. Brown, J. Cooperstein, and M. Prakash: Nucl. Phys. (in press).

23. S. Bruenn: Phys. Rev. Lett. $\underline{59}, 938$ (1987).

24. J. N. Bahcall and S. L. Glashow: Nature $\underline{326}, 376$ (1987);

J. N. Bahcall, T. Piran, W. H. Press, and D. N. Spergel: Phys. Rev. Lett. (submitted April 1987).

25. J. Lattimer: Proceedings of the XI Particle and Nuclei Conf. (Kyoto, Japan, April 1987);

A. Burrows and J. Lattimer: "Neutrinos from SN1987a" University of Arizona preprint (April 1987).

26. W. D. Arnett and J. L. Rosner: Phys. Rev. Lett. $\underline{58}, 1906$ (1987).

27. K. Sato and H. Suzuki: Phys. Rev. Lett. $\underline{58,2722 ~(1987) . ~}$

28. E. W. Kolb, A. J. Stebbins, and M. S. Turner: Phys. Rev. D (in press).

29. R. G. W. Robertson et al: Reported in Telemark IV (March 1987): $m_{\nu}<27 \mathrm{eV}$ (95\% cl);

M. Frischi et al: Phys. Lett. 173B, 485 (1986): $m_{\nu}<18 \mathrm{eV}(95 \% \mathrm{cl})$.

30. D. N. Schramm: Proceedings of XXII Recontre de Moriond (March 1987). 